\title{
Observer-Based Decentralized Control for Uncertain Interconnected Systems of Neutral Type
}

\author{
Heli Hu, ${ }^{1}$ Dan Zhao, ${ }^{2}$ and Qingling Zhang ${ }^{3}$ \\ ${ }^{1}$ Key Laboratory of Manufacturing Industrial Integrated Automation, Shenyang University, Shenyang 110044, China \\ ${ }^{2}$ Department of Fundamental Teaching, Shenyang Institute of Engineering, Shenyang 110136, China \\ ${ }^{3}$ Institute of Systems Science, Northeastern University, Shenyang, Liaoning 110004, China
}

Correspondence should be addressed to Heli Hu; huheli2002@yahoo.com.cn

Received 4 April 2013; Accepted 8 June 2013

Academic Editor: Rongni Yang

Copyright (C) 2013 Heli Hu et al. This is an open access article distributed under the Creative Commons Attribution License, which permits unrestricted use, distribution, and reproduction in any medium, provided the original work is properly cited.

\begin{abstract}
The observer-based decentralized control problem is investigated for a class of uncertain interconnected systems of neutral type. Using the singular value decomposition approach, a full-order observer is designed to guarantee the asymptotic stability of the error dynamic system. A novel mathematical technique is developed to solve this design problem. Sufficient condition for uncertain interconnected systems of neutral type to be asymptotic stable is established based on the singular value decomposition method. Furthermore, the desired gains of observer and controller are obtained by the explicit expressions in terms of some free parameters. Finally, an illustrative example is used to demonstrate the proposed approach, and the corresponding simulation results are given to elucidate the effectiveness.
\end{abstract}

\section{Introduction}

Nowadays the systems have become more and more large, and for the interconnected systems decentralized control has obvious advantage that overcomes the limitations of the traditional centralized control requiring sufficiently large communication bandwidth to exchange information between the subsystems. Therefore, the decentralized control scheme [111] only making use of local information available is very popular among the researchers and the engineers. In [1], an algorithm formulated within the convex optimization framework is proposed to investigate the strict dissipativity of the linear interconnected systems. A decentralized structure of dissipative state-feedback controllers is designed. In [2, 3], a decentralized adaptive output-feedback stabilizer and a decentralized $L_{1}$ adaptive controller are proposed to stabilize a class of large-scale nonlinear systems, respectively. Different from the constant delays involved in the considered system without a priori knowledge of subsystem high-frequencygain signs in [2], the interconnected nonlinearities and unmodeled dynamics are included in the considered systems in [3]. Based on the concepts of dynamic graphs and dynamic adjacency matrix, a modeling method of a complex dynamic interconnected system is considered in [4]. In [5], a decentralized dynamic output feedback based on linear controller is proposed to robust stabilize a class of nonlinear interconnected systems coupled by nonlinear interconnections that are unknown and quadratically bounded. However, neither constant delay nor time-varying delays are considered in $[4,5]$.

On the other hand, the neutral system is the general form of delay system that contains the same highest order derivatives for the state vector $x(t)$, at both time $t$ and past time(s) $t_{s} \leq t$. Many models of practical systems can be described by functional differential equation of neutral type [12]. Physical examples for neutral system have distributed networks [13], population ecology [14], heat exchangers, robots in contact with rigid environments [15], and so forth. In recent years, the stability analysis and robust control problems of neutral delay systems have been considered extensively (see, e.g., [16-23]). In [16-18], the stability problems of neutral systems are investigated. The difference among them is that Balasubramaniam et al. [16] focus on the stability of the neutral systems with both constant and timevarying delays using the method of nonuniformly dividing 
the whole delay interval into multiple segments, Rakkiyappan et al. [17] study the stability of the neutral systems with interval time-varying delays and nonlinear perturbations using the method of a new Lyapunov functional approach, and Nian et al. [18] deal with the stability of neutral systems with only a constant delay using the method of state matrix decomposition. However, the design problems of the control law have not been considered in them.

Furthermore, Ma et al. [19] develop a control method for neutral systems with a single input and some restrictions on the system matrices using a differential-difference inequality and the transformation technique. Han et al. [20] utilize a discretized Lyapunov-Krasovskii functional approach to investigate the stability of the linear neutral systems with small and nonsmall discrete delays. But its discrete application to control design yields nonlinear conditions, which may not be easily computable. In [21], the robust adaptive stabilization problem is investigated for neutral timedelay systems with uncertainties, and an adaptive scheme is introduced to estimate the bounds on uncertainties. But the matched condition is required for the disturbance vector of the considered system. It is worth pointing out that many researches on the neutral systems are often restricted to the stability analysis without controller or static state feedback control schemes.

Nowadays, observer control is also an attractive topic [24-28]. In [24], two controllers based on state feedback and observer output feedback are designed for networked systems with discrete and distributed delays subject to quantization and packet dropout. Also, a compensation scheme is proposed to deal with the effect of random packet dropout through communication network. In [25], Su et al. introduce a new model transformation for considered discrete-time TS fuzzy systems to realize the design of dynamic outputfeedback controller. Utilizing an approximation for timevarying delay state, a new comparison model is proposed. Furthermore, the $l_{2}-l_{\infty}$ filtering problem for a class of discrete-time T-S fuzzy systems with time-varying delays is studied in [26]. The anticipated full- and reduced-order filter design is cast into a convex optimization problem, which can be efficiently solved by standard numerical algorithms. In [27], an improved fuzzy observer design that has some advantages, such as the less conservatism and the satisfactory multiple performance required by the fault detection, is presented. Moreover, Messaoud et al. [28] propose a new design of functional unknown input observer for nonlinear systems. In particular, necessary and sufficient conditions for the existence of the anticipated observer are presented.

To the best of the authors' knowledge, the observer-based decentralized control problems of uncertain interconnected systems with neutral type have not yet been investigated, which motivates the present study. In this paper, the asymptotic stabilization of a class of uncertain neutral interconnected systems with time-varying delays in state, control input, and interconnections is made. In framework of linear matrix equalities, the design of the observer and controller is formulated. Sufficiently the influence of interconnections on the system performance is taken into account, and interconnections are dealt with by flexible techniques. These strategies allow one to obtain less conservative stabilization conditions. A numerical example and the corresponding simulation results are given to illustrate the effectiveness of the proposed method of decentralized controller based on observer.

The remainder of the paper is organized as follows. The observer-based decentralized control problem formulation is described in Section 2. In Section 3, the desired gains of observer and controller are obtained by the explicit expressions in terms of some free parameters. A numerical example and the corresponding simulation results are presented in Section 4. The conclusion is provided in Section 5.

\section{Problem Formulation}

Consider the following uncertain neutral interconnected systems composed of $N$ subsystems:

$$
\begin{aligned}
\dot{x}_{i}(t) & -A_{i \eta_{i}} \dot{x}_{i}\left(t-\eta_{i}(t)\right) \\
= & {\left[A_{i}+\Delta A_{i}(t)\right] x_{i}(t) } \\
& +\left[A_{i \sigma_{i}}+\Delta A_{i \sigma_{i}}(t)\right] x_{i}\left(t-\sigma_{i}(t)\right) \\
& +\left[B_{i}+\Delta B_{i}(t)\right] u_{i}(t) \\
& +\sum_{j=1, j \neq i}^{N}\left[A_{i j}+\Delta A_{i j}\right] x_{j}\left(t-\tau_{i j}(t)\right), \\
y_{i}(t)= & C_{i} x_{i}(t)+D_{i} u_{i}(t), \\
x_{i}(t) & =\phi_{i}(t), \quad t \in[-l, 0], i=1,2, \ldots, N,
\end{aligned}
$$

where $x_{i}(t) \in \mathfrak{R}^{n_{i}}, u_{i}(t) \in \mathfrak{R}^{m_{i}}$, and $y_{i}(t) \in \mathfrak{R}^{p_{i}}$ are the state, control input, and measurement output of the $i$ th subsystem, respectively. $A_{i}, A_{i \sigma_{i}}, A_{i \eta_{i}}, B_{i}, A_{i j}, C_{i}$, and $D_{i}$ are known constant matrices of appropriate dimensions. $\phi_{i}(t)$ is the initial condition. $\sigma_{i}(t), \eta_{i}(t)$, and $\tau_{i j}(t)$ are the timevarying delays. Assume that there exist constants $f_{i 0}, g_{i 0}, l_{i 0}$, $f_{i}, g_{i}, l_{i}$, and $l$ satisfying

$$
\begin{gathered}
0 \leq \sigma_{i}(t) \leq f_{i 0}, \quad 0 \leq \eta_{i}(t) \leq g_{i 0}, \quad 0 \leq \tau_{i j}(t) \leq l_{i 0}, \\
\dot{\sigma}_{i}(t) \leq f_{i}<1, \quad \dot{\eta}_{i}(t) \leq g_{i}<1, \quad \dot{\tau}_{i j}(t) \leq l_{i}<1, \\
l=\max \left\{f_{i 0}, g_{i 0}, l_{i 0}\right\}, \quad i, j=1,2 \ldots, N, \quad j \neq i .
\end{gathered}
$$

Time-varying parametric uncertainties $\Delta A_{i}(t), \Delta A_{i \sigma_{i}}(t)$, $\Delta B_{i}(t)$, and $\Delta A_{i j}(t)$ are assumed to satisfy

$$
\begin{gathered}
{\left[\begin{array}{cccc}
\Delta A_{i}(t) & \Delta A_{i \sigma_{i}}(t) & \Delta B_{i}(t) & \Delta A_{i j}(t)
\end{array}\right]} \\
\quad=M_{i} F_{i}(t)\left[\begin{array}{llll}
N_{i 1} & N_{i \sigma_{i}} & N_{i 2} & E_{i j}
\end{array}\right]
\end{gathered}
$$


where matrices $M_{i}, N_{i 1}, N_{i \sigma_{i}}, N_{i 2}$, and $E_{i j}$ are constant matrices of appropriate dimensions and $F_{i}(t)$ is the unknown matrix function satisfying

$$
F_{i}^{T}(t) F_{i}(t) \leq I, \quad \forall t \geq 0
$$

The following assumptions are made on the considered system (1).

Assumption 1. Suppose that the matrix $C_{i}$ has full row rank (i.e., $\left.\operatorname{rank}\left(C_{i}\right)=p_{i}\right)$. Then the singular value decomposition of $C_{i}$ presents as follows:

$$
C_{i}=U_{i}\left[\begin{array}{ll}
S_{i} & 0
\end{array}\right] V_{i}^{T},
$$

where $S_{i} \in \mathfrak{R}^{p_{i} \times p_{i}}$ is a diagonal matrix with positive elements in a decreasing order, $0 \in \mathfrak{R}^{p_{i} \times\left(n_{i}-p_{i}\right)}$ is a zero matrix, and $U_{i} \in \mathfrak{R}^{p_{i} \times p_{i}}$ and $V_{i} \in \mathfrak{R}^{n_{i} \times n_{i}}$ are unitary matrices.

Assumption 2. The matrix $A_{i \eta_{i}} \neq 0$ and $\left\|A_{i \eta_{i}}\right\|<1$.

Consider the following observer-based decentralized control for system (1):

$$
\begin{aligned}
\dot{\hat{x}}_{i}(t) & -A_{i \eta_{i}} \dot{\hat{x}}_{i}\left(t-\eta_{i}(t)\right) \\
= & A_{i} \widehat{x}_{i}(t)+B_{i} u_{i}(t)+A_{i \sigma_{i}} \widehat{x}_{i}\left(t-\sigma_{i}(t)\right) \\
& +\sum_{j=1, j \neq i}^{N} A_{i j} \widehat{x}_{j}\left(t-\tau_{i j}(t)\right)+L_{i}\left(y_{i}(t)-\widehat{y}_{i}(t)\right), \\
\widehat{y}_{i}(t) & =C_{i} \widehat{x}_{i}(t)+D_{i} u_{i}(t), \\
u_{i}(t) & =-K_{i} \widehat{x}_{i}(t)
\end{aligned}
$$

where $\widehat{x}_{i}(t) \in \mathfrak{R}^{n_{i}}$ and $\widehat{y}_{i}(t) \in \mathfrak{R}^{p_{i}}$ are the state and output vectors of the observer and $K_{i} \in \mathfrak{R}^{m_{i} \times n_{i}}$ and $L_{i} \in \mathfrak{R}^{n_{i} \times p_{i}}$ are the controller gain and observer gain to be designed. System (1) with the observer-based control (6) can be rewritten as

$$
\begin{aligned}
{\left[\begin{array}{c}
\dot{\hat{x}}_{i}(t) \\
\dot{e}_{i}(t)
\end{array}\right]=} & {\left[\begin{array}{cc}
A_{i}-B_{i} K_{i} & L_{i} C_{i} \\
0 & A_{i}-L_{i} C_{i}
\end{array}\right]\left[\begin{array}{l}
\hat{x}_{i}(t) \\
e_{i}(t)
\end{array}\right] } \\
& +\left[\begin{array}{cc}
A_{i \sigma_{i}} & 0 \\
0 & A_{i \sigma_{i}}
\end{array}\right]\left[\begin{array}{l}
\hat{x}_{i}\left(t-\sigma_{i}(t)\right) \\
e_{i}\left(t-\sigma_{i}(t)\right)
\end{array}\right] \\
& +\left[\begin{array}{cc}
A_{i \eta_{i}} & 0 \\
0 & A_{i \eta_{i}}
\end{array}\right]\left[\begin{array}{l}
\dot{\hat{x}}_{i}\left(t-\eta_{i}(t)\right) \\
\dot{e}_{i}\left(t-\eta_{i}(t)\right)
\end{array}\right] \\
& +\left[\begin{array}{c}
\sum_{j=1, j \neq i}^{N} A_{i j} \hat{x}_{j}\left(t-\tau_{i j}(t)\right) \\
\sum_{j=1, j \neq i}^{N} A_{i j} e_{j}\left(t-\tau_{i j}(t)\right)
\end{array}\right] \\
& +\left[\begin{array}{c}
0 \\
M_{i}
\end{array}\right] \Delta_{i}(t)
\end{aligned}
$$

where the signal $e_{i}(t)=x_{i}(t)-\widehat{x}_{i}(t)$ is defined as the estimated error of system, $\Delta_{i}(t)=F_{i}(t)\left[\left(N_{i 1}-N_{i 2} K_{i}\right) \widehat{x}_{i}(t)+\right.$ $N_{i 1} e_{i}+N_{i \sigma_{i}} \widehat{x}_{i \sigma_{i}}+N_{i \sigma_{i}} e_{i \sigma_{i}}+\sum_{j=1, j \neq i}^{N} E_{i j} \widehat{x}_{j}\left(t-\tau_{i j}(t)\right)+$ $\left.\sum_{j=1, j \neq i}^{N} E_{i j} \mathrm{e}_{j}\left(t-\tau_{i j}(t)\right)\right]$, and the uncertainty $\Delta_{i}(t)$ satisfies the following quadratic inequality:

$$
\Delta_{i}^{T}(t) \Delta_{i}(t) \leq \xi_{i}^{T}(t) \Theta_{i}^{T} \Theta_{i} \xi_{i}(t)
$$

where

$$
\begin{aligned}
& \xi_{i}^{T}(t)=\left[\begin{array}{llll}
\widehat{x}_{i}^{T}(t) & e_{i}^{T}(t) & \hat{x}_{i}^{T}\left(t-\sigma_{i}(t)\right) & e_{i}^{T}\left(t-\sigma_{i}(t)\right)
\end{array}\right. \\
& \dot{\hat{x}}_{i}^{T}\left(t-\eta_{i}(t)\right) \dot{e}_{i}^{T}\left(t-\eta_{i}(t)\right) \widehat{x}_{1}^{T}\left(t-\tau_{i 1}(t)\right) \\
& \cdots \widehat{x}_{i-1}^{T}\left(t-\tau_{i} \quad{ }_{i-1}(t)\right) \widehat{x}_{i+1}^{T}\left(t-\tau_{i} \quad{ }_{i+1}(t)\right) \\
& \cdots \quad \widehat{x}_{N}^{T}\left(t-\tau_{i N}(t)\right) e_{1}^{T}\left(t-\tau_{i 1}(t)\right) \\
& \cdots e_{i-1}^{T}\left(t-\tau_{i \quad i-1}(t)\right) \quad e_{i+1}^{T}\left(t-\tau_{i}{ }_{i+1}(t)\right) \\
& \left.\cdots \quad e_{N}^{T}\left(t-\tau_{i N}(t)\right)\right], \\
& \Theta_{i}=\left[\begin{array}{lllllll}
\left(N_{i 1}-N_{i 2} K_{i}\right) & N_{i 1} & N_{i \sigma_{i}} & N_{i \sigma_{i}} & 0 & 0 & E_{i 1}
\end{array}\right. \\
& \begin{array}{lllllll}
\cdots & E_{i-1} & E_{i+1} & \cdots & E_{i N} & E_{i 1}
\end{array} \\
& \left.\cdots E_{i-1} E_{i+1} \cdots E_{i N}\right] \text {. }
\end{aligned}
$$

Definition 3. Consider system (1) with the observer-based control (6). System (1) is said to be robustly stabilizable by the observer-based control (6), if the closed-loop system (7) satisfying Assumptions 1 and 2 is asymptotically stable.

Lemma 4 (see [29]). For a given $C_{i} \in \mathfrak{R}^{p_{i} \times n_{i}}$ with $\operatorname{rank}\left(C_{i}\right)=$ $p_{i}$, assume that $Q_{i 2} \in \mathfrak{R}^{n_{i} \times n_{i}}$ is a symmetric matrix; then there exists a matrix $\widehat{Q}_{i 2} \in \mathfrak{R}^{p_{i} \times p_{i}}$ such that $C_{i} Q_{i 2}=\widehat{Q}_{i 2} C_{i}$ if and only if

$$
Q_{i 2}=V_{i}\left[\begin{array}{cc}
\widehat{X}_{i 1} & 0 \\
0 & \widehat{X}_{i 2}
\end{array}\right] V_{i}^{T},
$$

where $\widehat{X}_{i 1} \in \mathfrak{R}^{p_{i} \times p_{i}}$ and $\widehat{X}_{i 2} \in \mathfrak{R}^{\left(n_{i}-p_{i}\right) \times\left(n_{i}-p_{i}\right)}$.

\section{Main Result}

Theorem 5. Consider uncertain neutral interconnected systems (1) with (2), (3), and (6). If there exists a solution $Q_{i k}>$ $0(k=1,3,4,5,6), \widehat{X}_{i 1}>0, \widehat{X}_{i 2}>0, \bar{W}_{j i}>0, \bar{G}_{j i}>0, W_{i j}>$ $0, G_{i j}>0, R_{i 1}$, and $R_{i 2}$ such that the following inequalities hold:

$$
\Omega_{i}<0
$$


then system (1) is robustly stabilizable by the observer-based where control (6) with $K_{i}=R_{i 1} Q_{i 1}^{-1}$ and $L_{i}=R_{i 2} U_{i} S_{i} \widehat{X}_{i 1}^{-1} S_{i}^{-1} U_{i}^{T}$,

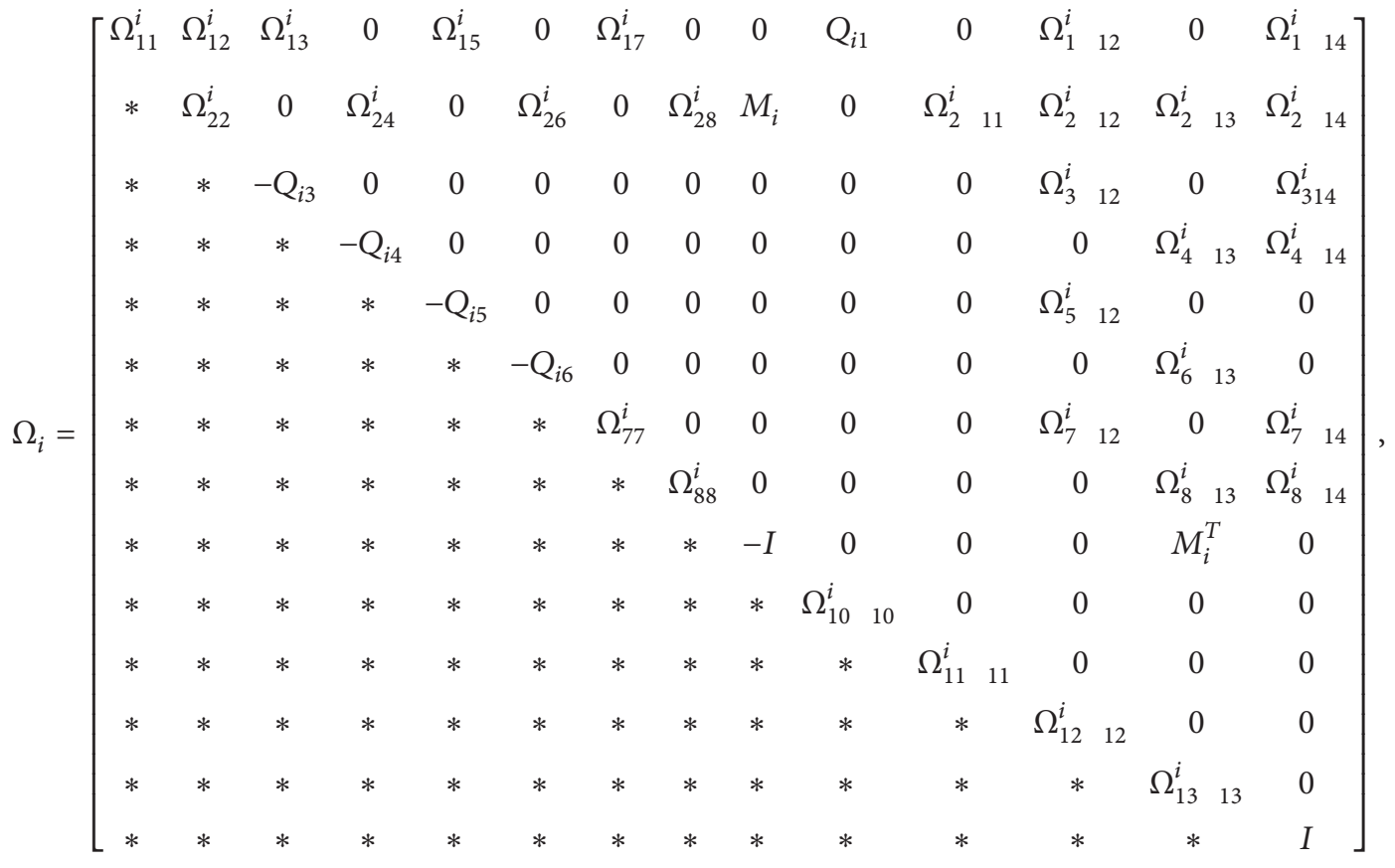

$$
\begin{aligned}
& \Omega_{11}^{i}=A_{i} Q_{i 1}+Q_{i 1} A_{i}^{T}-B_{i} R_{i 1}-R_{i 1}^{T} B_{i}^{T}+\sum_{j=1, j \neq i}^{N} \frac{1}{1-l_{j}} \bar{W}_{j i}, \quad \Omega_{12}^{i}=R_{i 2} C_{i}, \quad \Omega_{13}^{i}=A_{i \sigma_{i}} Q_{i 3}, \\
& \Omega_{15}^{i}=A_{i \eta_{i}} \mathrm{Q}_{i 5}, \quad \Omega_{1}^{i}{ }_{12}=Q_{i 1} A_{i}^{T}-R_{i 1}^{T} B_{i}^{T}, \quad \Omega_{17}^{i}=\left[\begin{array}{lllllll}
A_{i 1} & \cdots & A_{i} i-1 & A_{i} & & \cdots & A_{i N}
\end{array}\right], \\
& \Omega_{114}^{i}=Q_{i 1} N_{i 1}^{T}-R_{i 1}^{T} N_{i 2}^{T}, \quad \Omega_{24}^{i}=A_{i \sigma_{i}} Q_{i 4}, \quad \Omega_{22}^{i}=A_{i} Q_{i 2}+Q_{i 2} A_{i}^{T}-R_{i 2} C_{i}-C_{i}^{T} R_{i 2}^{T}+\sum_{j=1, j \neq i}^{N} \frac{1}{1-l_{j}} \bar{G}_{j i}, \\
& \Omega_{26}^{i}=A_{i \eta_{i}} Q_{i 6}, \quad \Omega_{28}^{i}=\Omega_{17}^{i}, \quad \Omega_{211}^{i}=Q_{i 2}, \quad \Omega_{2}^{i}{ }_{12}=C_{i}^{T} R_{i 2}^{T}, \\
& \Omega_{214}^{i}{ }_{14}=Q_{i 2} N_{i 1}^{T}, \quad \Omega_{213}^{i}=Q_{i 2} A_{i}^{T}-C_{i}^{T} R_{i 2}^{T}, \quad \Omega_{312}^{i}=Q_{i 3} A_{i \sigma_{i}}^{T}, \quad \Omega_{314}^{i}=Q_{i 3} N_{i \sigma_{i}}^{T}, \\
& \Omega_{413}^{i}=Q_{i 4} A_{i \sigma_{i}}^{T}, \quad \Omega_{613}^{i}=Q_{i 6} A_{i \eta_{i},}^{T}, \quad \Omega_{414}^{i}{ }_{14}=Q_{i 4} N_{i \sigma_{i}}^{T}, \quad \Omega_{512}^{i}=Q_{i 5} A_{i \eta_{i}}^{T}, \\
& \Omega_{77}^{i}=\operatorname{diag}\left\{-W_{i 1}, \ldots,-W_{i}{ }_{i-1},-W_{i}{ }_{i+1}, \ldots,-W_{i N}\right\}, \quad \Omega_{7}^{i}{ }_{12}=\left(\Omega_{17}^{i}\right)^{T}, \quad \Omega_{8}^{i} \quad{ }_{13}=\Omega_{7}^{i} \quad{ }_{12}, \\
& \Omega_{7}^{i} \quad{ }_{14}=\left[\begin{array}{llllllll}
E_{i 1} & \cdots & E_{i} i_{i-1} & E_{i} & { }_{i+1} & \cdots & E_{i N}
\end{array}\right]^{T}, \quad \Omega_{8}^{i} \quad{ }_{14}=\Omega_{7}^{i} \quad{ }_{14}, \\
& \Omega_{88}^{i}=\operatorname{diag}\left\{-G_{i 1}, \ldots,-G_{i}{ }_{i-1},-G_{i}{ }_{i+1}, \ldots,-G_{i N}\right\}, \quad \Omega_{10}^{i} \quad{ }_{10}=-\left(1-f_{i}\right) Q_{i 3}, \quad \Omega_{11}^{i}{ }_{11}=-\left(1-f_{i}\right) Q_{i 4}, \\
& \Omega_{12 \quad 12}^{i}=-\left(1-g_{i}\right) Q_{i 5}, \quad \Omega_{13}^{i} \quad 13=-\left(1-g_{i}\right) Q_{i 6}, \quad \bar{W}_{j i}=Q_{i 1} W_{j i} Q_{i 1}, \quad \bar{G}_{j i}=Q_{i 1} G_{j i} Q_{i 1}, \\
& Q_{i 2}=V_{i}\left[\begin{array}{cc}
\widehat{X}_{i 1} & 0 \\
0 & \widehat{X}_{i 2}
\end{array}\right] V_{i}^{T}, \quad i, j=1,2 \ldots, N, j \neq i .
\end{aligned}
$$

Proof. According to Schur complement, inequality (11) is equivalent to the following inequality: 


$$
\begin{aligned}
\Gamma_{i 1}= & {\left[\begin{array}{ccccccccc}
\Omega_{11}^{i} & \Omega_{12}^{i} & \Omega_{13}^{i} & 0 & \Omega_{15}^{i} & 0 & \Omega_{17}^{i} & 0 & 0 \\
* & \Omega_{22}^{i} & 0 & \Omega_{24}^{i} & 0 & \Omega_{26}^{i} & 0 & \Omega_{28}^{i} & M_{i} \\
* & * & -Q_{i 3} & 0 & 0 & 0 & 0 & 0 & 0 \\
* & * & * & -Q_{i 4} & 0 & 0 & 0 & 0 & 0 \\
* & * & * & * & -Q_{i 5} & 0 & 0 & 0 & 0 \\
* & * & * & * & * & -Q_{i 6} & 0 & 0 & 0 \\
* & * & * & * & * & * & \Omega_{77}^{i} & 0 & 0 \\
* & * & * & * & * & * & * & \Omega_{88}^{i} & 0 \\
* & * & * & * & * & * & * & * & -I
\end{array}\right] } \\
& +\Upsilon_{i 1}^{T}\left(\frac{1}{1-f_{i}} Q_{i 3}^{-1}\right) \Upsilon_{i 1}+\Upsilon_{i 2}^{T}\left(\frac{1}{1-f_{i}} Q_{i 4}^{-1}\right) \Upsilon_{i 2}+\Upsilon_{i 3}^{T}\left(\frac{1}{1-g_{i}} Q_{i 5}^{-1}\right) \Upsilon_{i 3}+\Upsilon_{i 4}^{T}\left(\frac{1}{1-g_{i}} Q_{i 6}^{-1}\right) \Upsilon_{i 4}+\Upsilon_{i 5}^{T} \Upsilon_{i 5}<0,
\end{aligned}
$$

where

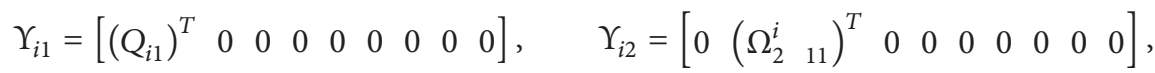

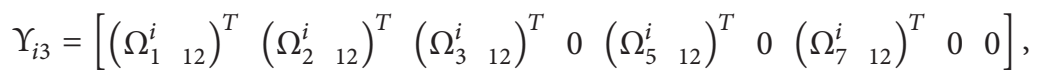

$$
\begin{aligned}
& \Upsilon_{i 4}=\left[\begin{array}{lll}
0 & \left(\begin{array}{ll}
\Omega_{2}^{i} & 13
\end{array}\right)^{T} \quad 0 \quad\left(\begin{array}{ll}
\Omega_{4}^{i} & 13
\end{array}\right)^{T} 0\left(\begin{array}{ll}
\Omega_{6}^{i} & 13
\end{array}\right)^{T} 0\left(\begin{array}{ll}
\Omega_{8}^{i} & 13
\end{array}\right)^{T} M_{i}
\end{array}\right],
\end{aligned}
$$

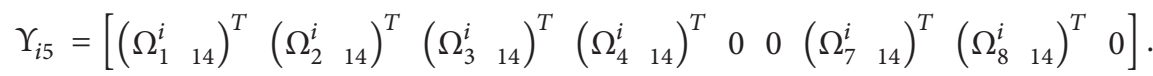

By Lemma 4, the conditions $Q_{i 2}=V_{i}\left[\begin{array}{cc}\widehat{X}_{i 1} & 0 \\ 0 & \widehat{X}_{i 2}\end{array}\right] V_{i}^{T}$ and $\widehat{Q}_{i 2}=U_{i} S_{i} \widehat{X}_{i 1} S_{i}^{-1} U_{i}^{T}$ imply the condition $C_{i} Q_{i 2}=\widehat{Q}_{i 2} C_{i}$. In view of $K_{i}=R_{i 1} Q_{i 1}^{-1}, L_{i}=R_{i 2} U_{i} S_{i} \widehat{X}_{i 1}^{-1} S_{i}^{-1} U_{i}^{T}, \bar{W}_{j i}=$
$Q_{i 1} W_{j i} Q_{i 1}$, and $\bar{G}_{j i}=Q_{i 1} G_{j i} Q_{i 1}$, the matrix inequality (13) is equivalent to

$$
\begin{aligned}
\Gamma_{i 2}= & {\left[\begin{array}{ccccccccc}
\Xi_{11}^{i} & \Xi_{12}^{i} & \Xi_{13}^{i} & 0 & \Xi_{15}^{i} & 0 & \Omega_{17}^{i} & 0 & 0 \\
* & \Xi_{22}^{i} & 0 & \Xi_{24}^{i} & 0 & \Xi_{26}^{i} & 0 & \Omega_{18}^{i} & M_{i} \\
* & * & -Q_{i 3} & 0 & 0 & 0 & 0 & 0 & 0 \\
* & * & * & -Q_{i 4} & 0 & 0 & 0 & 0 & 0 \\
* & * & * & * & -Q_{i 5} & 0 & 0 & 0 & 0 \\
* & * & * & * & * & -Q_{i 6} & 0 & 0 & 0 \\
* & * & * & * & * & * & \Omega_{77}^{i} & 0 & 0 \\
* & * & * & * & * & * & * & \Omega_{88}^{i} & 0 \\
* & * & * & * & * & * & * & * & -I
\end{array}\right]+\Lambda_{i}^{T}\left(\frac{1}{1-g_{i}} Q_{i 5}^{-1}\right) \Lambda_{i}+\widehat{\Lambda}_{i}^{T}\left(\frac{1}{1-g_{i}} Q_{i 6}^{-1}\right) \widehat{\Lambda}_{i}+\bar{\Lambda}_{i}^{T} \bar{\Lambda}_{i} } \\
& +\left[\begin{array}{c}
Q_{i 1} \\
0 \\
0 \\
0 \\
0 \\
0 \\
0 \\
0 \\
0
\end{array}\right]\left(\frac{1}{1-f_{i}} Q_{i 3}^{-1}\right)\left[\begin{array}{c}
0 \\
0 \\
0 \\
0 \\
0 \\
0 \\
0 \\
0
\end{array}\right]+\left[\begin{array}{c}
Q_{i 2} \\
0 \\
0 \\
0 \\
0 \\
0 \\
0 \\
0
\end{array}\right]\left(\frac{1}{1-f_{i}} Q_{i 4}^{-1}\right)\left[\begin{array}{c}
0 \\
Q_{i 2} \\
0 \\
0 \\
0 \\
0 \\
0 \\
0 \\
0
\end{array}\right]<0,
\end{aligned}
$$


where

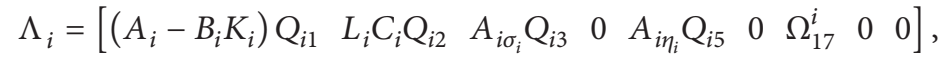

$$
\begin{aligned}
& \widehat{\Lambda}_{i}=\left[\begin{array}{lllllllll}
0 & \left(A_{i}-L_{i} C_{i}\right) Q_{i 2} & 0 & A_{i \sigma_{i}} Q_{i 4} & 0 & A_{i \eta_{i}} Q_{i 6} & 0 & \Omega_{17}^{i} & M_{i}
\end{array}\right], \\
& \bar{\Lambda}_{i}=\left[\begin{array}{lllllllll}
\left(N_{i 1}-N_{i 2} K_{i}\right) Q_{i 1} & N_{i 1} Q_{i 2} & N_{i \sigma_{i}} Q_{i 3} & N_{i \sigma_{i}} Q_{i 4} & 0 & 0 & \left(\begin{array}{ll}
\Omega_{7}^{i} & 14
\end{array}\right)^{T} & \left(\begin{array}{ll}
\Omega_{8}^{i} & 14
\end{array}\right)^{T} & 0
\end{array}\right] \text {, } \\
& \Xi_{11}^{i}=Q_{i 1}\left(A_{i}-B_{i} K_{i}\right)^{T}+\left(A_{i}-B_{i} K_{i}\right) Q_{i 1}+\sum_{j=1, j \neq i}^{N} \frac{1}{1-l_{j}} \bar{W}_{j i}, \quad \Xi_{12}^{i}=L_{i} C_{i} Q_{i 2}, \quad \Xi_{13}^{i}=A_{i \sigma_{i}} Q_{i 3}, \\
& \Xi_{15}^{i}=A_{i \eta_{i}} Q_{i 5}, \quad \Xi_{22}^{i}=Q_{i 2}\left(\mathrm{~A}_{i}-L_{i} C_{i}\right)^{T}+\left(A_{i}-L_{i} C_{i}\right) Q_{i 2}+\sum_{j=1, j \neq i}^{N} \frac{1}{1-l_{j}} \bar{G}_{j i}, \quad \Xi_{24}^{i}=A_{i \sigma_{i}} Q_{i 4}, \\
& \Xi_{26}^{i}=A_{i \eta_{i}} Q_{i 6}
\end{aligned}
$$
where

Pre- and postmultiplying matrix $\Gamma_{i 2}$ in (15) by $\Pi_{i}^{T}$ and $\Pi_{i}$,

$$
\begin{aligned}
\Pi_{i} & =\operatorname{diag}\left(Q_{i 1}^{-1}, Q_{i 2}^{-1}, Q_{i 3}^{-1}, Q_{i 4}^{-1}, Q_{i 5}^{-1}, Q_{i 6}^{-1}, I, I, I\right) \\
& =\operatorname{diag}\left(P_{i 1}, P_{i 2}, P_{i 3}, P_{i 4}, P_{i 5}, P_{i 6}, I, I, I\right)>0,
\end{aligned}
$$

we obtain

$\Gamma_{i 3}$

$$
\begin{aligned}
& {\left[\begin{array}{ccccccccc}
\Psi_{11}^{i} & \Psi_{12}^{i} & \Psi_{13}^{i} & 0 & \Psi_{15}^{i} & 0 & \Psi_{17}^{i} & 0 & 0 \\
* & \Psi_{22}^{i} & 0 & \Psi_{24}^{i} & 0 & \Psi_{26}^{i} & 0 & \Psi_{28}^{i} & P_{i 2} M_{i} \\
* & * & -Q_{i 3} & 0 & 0 & 0 & 0 & 0 & 0 \\
* & * & * & -Q_{i 4} & 0 & 0 & 0 & 0 & 0 \\
* & * & * & * & -Q_{i 5} & 0 & 0 & 0 & 0 \\
* & * & * & * & * & -Q_{i 6} & 0 & 0 & 0 \\
* & * & * & * & * & * & \Psi_{77}^{i} & 0 & 0 \\
* & * & * & * & * & * & * & \Psi_{88}^{i} & 0 \\
* & * & * & * & * & * & * & * & -I
\end{array}\right] } \\
& +\Phi_{i}^{T}\left(\frac{1}{1-g_{i}} P_{i 5}\right) \Phi_{i}+\widehat{\Phi}_{i}^{T}\left(\frac{1}{1-g_{i}} P_{i 6}\right) \widehat{\Phi}_{i} \\
& +\bar{\Phi}_{i}^{T} \bar{\Phi}_{i}<0
\end{aligned}
$$

where

$$
\begin{aligned}
\Phi_{i} & =\left[\begin{array}{llllllllll}
A_{i}-B_{i} K_{i} & L_{i} C_{i} & A_{i \sigma_{i}} & 0 & A_{i \eta_{i}} & 0 & \Omega_{17}^{i} & 0 & 0
\end{array}\right], \\
\widehat{\Phi}_{i} & =\left[\begin{array}{lllllllll}
0 & A_{i}-L_{i} C_{i} & 0 & A_{i \sigma_{i}} & 0 & A_{i \eta_{i}} & 0 & \Omega_{17}^{i} & M_{i}
\end{array}\right],
\end{aligned}
$$

$\bar{\Phi}_{i}$

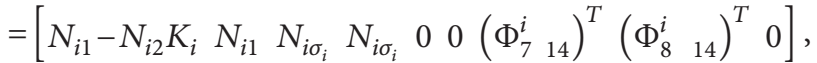

$$
\begin{aligned}
& \Psi_{11}^{i}=\left(A_{i}-B_{i} K_{i}\right)^{T} P_{i 1}+P_{i 1}\left(A_{i}-B_{i} K_{i}\right) \\
& +\sum_{j=1, j \neq i}^{N} \frac{1}{1-l_{j}} \bar{W}_{j i}+\frac{1}{1-f_{i}} P_{i 3}, \\
& \Psi_{12}^{i}=P_{i 1} L_{i} C_{i}, \quad \Psi_{13}^{i}=P_{i 1} A_{i \sigma_{i}}, \\
& \Psi_{15}^{i}=P_{i 1} A_{i \eta_{i}}, \quad \Psi_{17}^{i}=P_{i 1} \Omega_{17}^{i}, \\
& \Psi_{24}^{i}=P_{i 2} A_{i \sigma_{i}}, \quad \Psi_{26}^{i}=P_{i 2} A_{i \eta_{i}}, \\
& \Psi_{22}^{i}=\left(A_{i}-L_{i} C_{i}\right)^{T} P_{i 2}+P_{i 2}\left(A_{i}-L_{i} C_{i}\right) \\
& +\sum_{j=1, j \neq i}^{N} \frac{1}{1-l_{j}} \bar{G}_{j i}+\frac{1}{1-f_{i}} P_{i 4}, \\
& \Psi_{28}^{i}=P_{i 2} \Omega_{17}^{i} .
\end{aligned}
$$

Construct the following Lyapunov functional candidate:

$$
\begin{aligned}
& V\left(\hat{x}_{t}, e_{t}\right) \\
& =\sum_{i=1}^{N}\left\{\widehat{x}_{i}^{T}(t) P_{i 1} \widehat{x}_{i}(t)+e_{i}^{T}(t) P_{i 2} e_{i}(t)\right. \\
& \quad+\frac{1}{1-f_{i}} \\
& \quad \times \int_{t-\sigma_{i}(t)}^{t}\left[\hat{x}_{i}^{T}(s) P_{i 3} \widehat{x}_{i}(s)+e_{i}^{T}(s) P_{i 4} e_{i}(s)\right] d s
\end{aligned}
$$




$$
\begin{aligned}
& +\frac{1}{1-g_{i}} \\
& \times \int_{t-\eta_{i}(t)}^{t}\left[\dot{\hat{x}}_{i}^{T}(s) P_{i 5} \dot{\hat{x}}_{i}(s)+\dot{e}_{i}^{T}(s) P_{i 6} \dot{e}_{i}(s)\right] d s \\
& +\frac{1}{1-l_{i}} \\
& \times \sum_{j=1, j \neq i}^{N} \int_{t-\tau_{i j}(t)}^{t}\left[\widehat{x}_{j}^{T}(s) W_{i j} \widehat{x}_{j}(s)\right. \\
& \left.\left.+e_{j}^{T}(s) G_{i j} e_{j}(s)\right] d s\right\} .
\end{aligned}
$$

For the system (1), the following structural identity holds:

$$
\begin{aligned}
\sum_{i=1}^{N} & \frac{1}{1-l_{i}} \sum_{j=1, j \neq i}^{N} x_{j}^{T}(t) W_{i j} x_{j}(t) \\
& =\sum_{i=1}^{N} x_{i}^{T}(t) \sum_{j=1, j \neq i}^{N} \frac{1}{1-l_{j}} W_{j i} x_{i}(t) .
\end{aligned}
$$

By some simple derivations, the time derivative of $V\left(\widehat{x}_{t}, e_{t}\right)$ along the trajectories of (7) satisfies the following inequality:

$$
\dot{V}\left(\widehat{x}_{t}, e_{t}\right) \leq \sum_{i=1}^{N} \zeta_{i}^{T}(t) \Gamma_{i 3} \zeta_{i}(t)
$$

where

$$
\zeta_{i}^{T}(t)=\left[\begin{array}{ll}
\xi_{i}^{T}(t) & \Delta_{i}^{T}(t)
\end{array}\right]
$$

According to conditions (18) and (22), one can obtain that, for all $\zeta_{i} \neq 0$,

$$
\dot{V}\left(\widehat{x}_{t}, e_{t}\right)<0 \text {. }
$$

In addition, Assumption 2 guarantees system (1) is Lipschitzian in the term $\dot{x}_{i}\left(t-\eta_{i}(t)\right)$ with Lipschitz constant less than 1 [12]. Therefore, by Definition 3 with conditions (20) and (24), system (7) is asymptotically stable and system (1) is robustly stabilizable by observer-based control (6). This completes the proof.

Remark 6. One of the distinctive features of this paper is that the $N$ inequalities are included in (11), and only one is LMI due to the existence of the interconnected matrix variables $\bar{W}_{j i}=Q_{i 1} W_{j i} Q_{i 1}, W_{i j}, \bar{G}_{j i}=Q_{i 2} G_{j i} Q_{i 2}$, and $G_{i j}$ among $N$ inequalities. When $i=1$, the corresponding equality $\Omega_{1}<0$ in (11) is an LMI. Under the solvable condition of $\Omega_{1}<0$, we can apply Schur complement formula to the second inequality $\Omega_{2}<0$ in order to obtain the solvable LMI. The process is repeated until the last inequality. Thus, the controller gain $K_{i}$ and observer gain $L_{i}$ can be obtained by finding feasible set to $\Omega_{i}<0$ with feasp in [30].

\section{Illustrative Example}

Consider system (1) composed of two three-order subsystems with the following parameters:

$$
\begin{aligned}
& A_{1}=\left[\begin{array}{ccc}
-7.1 & 1.2 & 2.5 \\
-3.8 & -2.1 & 0.4 \\
2.1 & -3.1 & -5.4
\end{array}\right] \\
& A_{1 \sigma_{1}}=\left[\begin{array}{ccc}
-0.2 & -0.1 & -0.2 \\
0.2 & 0.1 & 0.1 \\
0.1 & -0.2 & -0.1
\end{array}\right] \\
& B_{1}=\left[\begin{array}{cc}
-2.9 & -1.3 \\
-0.3 & 2.5 \\
2.7 & -2.2
\end{array}\right], \quad A_{1 \eta_{1}}=\left[\begin{array}{ccc}
-0.2 & 0.1 & 0.2 \\
0.2 & -0.3 & 0.2 \\
0.1 & -0.2 & -0.1
\end{array}\right] \text {, } \\
& M_{1}=\left[\begin{array}{ccc}
-0.2 & -0.1 & -0.4 \\
0.6 & -0.2 & 0.2 \\
-0.1 & -0.3 & 0.3
\end{array}\right], \quad N_{12}=\left[\begin{array}{cc}
-0.1 & 0.3 \\
0.2 & -0.5 \\
0.1 & 0.2
\end{array}\right] \text {, } \\
& A_{12}=\left[\begin{array}{ccc}
-2.2 & -1.5 & 1.1 \\
-1.1 & 1.3 & 0.2 \\
0.3 & 1.1 & -0.5
\end{array}\right], \quad N_{11}=\left[\begin{array}{ccc}
0.2 & 0.7 & 0.3 \\
0.2 & -0.5 & -0.2 \\
0.5 & -0.1 & -0.1
\end{array}\right] \text {, } \\
& D_{1}=\left[\begin{array}{cc}
-1.4 & 0.4 \\
1.1 & -1.1
\end{array}\right], \quad N_{1 \sigma_{1}}=\left[\begin{array}{ccc}
0.1 & -0.1 & -0.3 \\
0.2 & -0.8 & -0.2 \\
-0.5 & 0.1 & 0.1
\end{array}\right] \text {, } \\
& E_{12}=\left[\begin{array}{ccc}
0.3 & 0.4 & 0.2 \\
-0.3 & -0.3 & -0.1 \\
-0.1 & 0.2 & 0.1
\end{array}\right], \quad \sigma_{1}(t)=0.1(2+\sin (t)) \text {, } \\
& C_{1}=\left[\begin{array}{ccc}
-2.3 & 3.9 & 1.2 \\
0.2 & 2.3 & -2.1
\end{array}\right], \quad \eta_{1}(t)=0.2(1+\cos (t)) \text {, } \\
& \tau_{12}(t)=0.1(1+\cos (t)), \quad A_{2}=\left[\begin{array}{ccc}
-7.6 & 2.1 & 1.3 \\
-2.1 & -1.5 & -2.1 \\
1.2 & -0.5 & -5.3
\end{array}\right] \text {, } \\
& A_{2 \sigma_{2}}=\left[\begin{array}{ccc}
0.1 & -0.1 & -0.2 \\
-0.2 & 0.3 & 0.1 \\
0.2 & 0.3 & 0.2
\end{array}\right], \quad B_{2}=\left[\begin{array}{cc}
-2.1 & 1.8 \\
-4.1 & 0.2 \\
2.1 & -2.1
\end{array}\right] \text {, } \\
& A_{2 \eta_{2}}=\left[\begin{array}{ccc}
0.1 & 0.1 & -0.1 \\
0.3 & 0.2 & 0.4 \\
0.1 & 0.2 & 0.1
\end{array}\right], \quad A_{21}=\left[\begin{array}{ccc}
-0.1 & 0.3 & -0.1 \\
0.1 & -0.2 & 0.2 \\
0.1 & -0.1 & 0.1
\end{array}\right] \text {, } \\
& M_{2}=\left[\begin{array}{ccc}
0.5 & -0.1 & 0.3 \\
-0.2 & -0.1 & 0.1 \\
-0.2 & 0.3 & 0.1
\end{array}\right], \quad N_{21}=\left[\begin{array}{ccc}
-0.1 & 0.2 & 0.1 \\
-0.1 & -0.3 & 0.5 \\
-0.2 & 0.1 & -0.5
\end{array}\right] \text {, } \\
& N_{2 \sigma_{2}}=\left[\begin{array}{ccc}
0.2 & -0.1 & 0.1 \\
0.3 & 0.2 & 0.2 \\
-0.2 & 0.1 & -0.1
\end{array}\right], \quad N_{22}=\left[\begin{array}{cc}
0.3 & 0.1 \\
-0.2 & -0.2 \\
0.1 & 0.3
\end{array}\right] \text {, } \\
& E_{21}=\left[\begin{array}{ccc}
-0.2 & 0.2 & -0.1 \\
0.1 & 0.1 & 0.2 \\
-0.1 & -0.2 & 0.1
\end{array}\right], \quad C_{2}=\left[\begin{array}{ccc}
-4.5 & 0.1 & 0.1 \\
0.1 & 0.3 & -0.2
\end{array}\right] \text {, } \\
& D_{2}=\left[\begin{array}{cc}
0.17 & 0.31 \\
-0.12 & 0.23
\end{array}\right], \quad \sigma_{2}(t)=0.2(1+\cos (t)) \text {, }
\end{aligned}
$$


$\eta_{2}(t)=0.1(2+\cos (t)), \quad \tau_{21}(t)=0.2(2+\sin (t))$.

Applying Matlab toolbox to solving inequality (11), we obtain the following results:

$$
\begin{aligned}
& Q_{11}=\left[\begin{array}{ccc}
1.4865 & 0.7955 & 0.4231 \\
0.7955 & 2.2754 & -1.5140 \\
0.4231 & -1.5140 & 5.2053
\end{array}\right], \\
& Q_{13}=\left[\begin{array}{ccc}
1.4324 & 0.2646 & 0.8637 \\
0.2646 & 1.1728 & -1.3599 \\
0.8637 & -1.3599 & 5.0693
\end{array}\right] \text {, } \\
& Q_{14}=\left[\begin{array}{ccc}
1.4285 & 0.2413 & 0.6513 \\
0.2413 & 1.0183 & -1.1782 \\
0.6513 & -1.1782 & 4.2542
\end{array}\right] \text {, } \\
& Q_{15}=\left[\begin{array}{ccc}
47.9235 & 6.6463 & -2.8148 \\
6.6463 & 44.1028 & 1.5746 \\
-2.8148 & 1.5746 & 43.3414
\end{array}\right] \text {, } \\
& Q_{16}=\left[\begin{array}{ccc}
40.1902 & 9.0430 & -2.8492 \\
9.0430 & 39.5447 & 2.1458 \\
-2.8492 & 2.1458 & 41.1207
\end{array}\right] \text {, } \\
& K_{1}=\left[\begin{array}{ccc}
1.4531 & -2.1903 & -0.7468 \\
-1.4570 & 2.4554 & 0.6993
\end{array}\right] \text {, } \\
& \widehat{Q}_{12}=\left[\begin{array}{cc}
0.5747 & 0.2102 \\
-0.7420 & 3.6344
\end{array}\right] \text {, } \\
& \widehat{X}_{11}=\left[\begin{array}{cc}
0.8267 & -0.7426 \\
-0.7426 & 3.3824
\end{array}\right], \quad \widehat{X}_{12}=2.0431 \text {, } \\
& \bar{W}_{21}=\left[\begin{array}{ccc}
6.6256 & 0.8468 & -2.8166 \\
0.8468 & 3.7842 & -1.9443 \\
-2.8166 & -1.9443 & 7.7792
\end{array}\right] \text {, } \\
& W_{21}=\left[\begin{array}{ccc}
8.0860 & -4.9568 & -2.7975 \\
-4.9568 & 3.9197 & 1.9259 \\
-2.7975 & 1.9259 & 1.2428
\end{array}\right] \text {, } \\
& \bar{G}_{21}=\left[\begin{array}{ccc}
3.6329 & 1.1769 & -1.7276 \\
1.1769 & 3.4642 & -1.9414 \\
-1.7276 & -1.9414 & 7.6914
\end{array}\right], \\
& G_{21}=\left[\begin{array}{ccc}
2.2006 & -2.2199 & -1.0134 \\
-2.2199 & 4.2063 & 1.6203 \\
-1.0134 & 1.6203 & 1.4176
\end{array}\right] \text {, } \\
& R_{11}=\left[\begin{array}{ccc}
-5.3350 & 3.3676 & -0.9899 \\
-1.5843 & 4.0258 & 0.3958
\end{array}\right]
\end{aligned}
$$$$
R_{12}=\left[\begin{array}{cc}
-1.2497 & -0.6324 \\
1.4433 & 0.6711 \\
0.6835 & -0.8005
\end{array}\right]
$$$$
W_{12}=\left[\begin{array}{ccc}
32.5716 & -0.1560 & 0.7872 \\
-0.1560 & 33.0680 & 1.2248 \\
0.7872 & 1.2248 & 32.6264
\end{array}\right] \text {, }
$$$$
G_{12}=\left[\begin{array}{ccc}
33.1699 & 0.4384 & -0.0119 \\
0.4384 & 33.4629 & 0.6883 \\
-0.0119 & 0.6883 & 33.2668
\end{array}\right] \text {, }
$$$$
L_{1}=\left[\begin{array}{cc}
-2.2325 & -0.0449 \\
2.5588 & 0.0366 \\
0.8420 & -0.2690
\end{array}\right]
$$$$
Q_{21}=\left[\begin{array}{ccc}
0.2476 & -0.0193 & -0.1246 \\
-0.0193 & 0.1848 & -0.0060 \\
-0.1246 & -0.0060 & 0.2346
\end{array}\right] \text {, }
$$$$
\widehat{Q}_{22}=\left[\begin{array}{cc}
0.1239 & -0.0000 \\
-0.0000 & 0.1239
\end{array}\right] \text {, }
$$$$
\widehat{\mathrm{X}}_{21}=\left[\begin{array}{cc}
0.1239 & -0.0005 \\
-0.0005 & 0.1197
\end{array}\right] \text {, }
$$$$
Q_{23}=\left[\begin{array}{ccc}
1.3638 & 0.0541 & -2.6550 \\
0.0541 & 2.8900 & -0.9487 \\
-2.6550 & -0.9487 & 11.4203
\end{array}\right] \text {, }
$$$$
R_{22}=\left[\begin{array}{cc}
-0.0336 & 1.5912 \\
0.0797 & 5.7463 \\
0.0145 & -2.5460
\end{array}\right] \text {, }
$$$$
Q_{24}=\left[\begin{array}{ccc}
1.8054 & 1.0651 & -3.0558 \\
1.0651 & 1.7394 & -3.5831 \\
-3.0558 & -3.5831 & 9.2685
\end{array}\right] \text {, }
$$$$
K_{2}=\left[\begin{array}{lll}
0.5773 & -8.9195 & -3.6070 \\
3.8724 & -6.5449 & -12.5895
\end{array}\right] \text {, }
$$$$
Q_{25}=\left[\begin{array}{ccc}
416.8897 & -302.9290 & -158.6713 \\
-302.9290 & 231.3825 & 118.1184 \\
-158.6713 & 118.1184 & 79.4590
\end{array}\right] \text {, }
$$$$
\widehat{X}_{22}=0.1013 \text {, }
$$$$
Q_{26}=\left[\begin{array}{ccc}
139.5613 & -92.2506 & -33.9039 \\
-92.2506 & 66.7926 & 18.6639 \\
-33.9039 & 18.6639 & 12.5276
\end{array}\right] \text {, }
$$$$
R_{21}=\left[\begin{array}{lll}
0.7642 & -1.6381 & -0.8647 \\
2.6539 & -1.2087 & -3.3972
\end{array}\right] \text {, }
$$$$
L_{2}=\left[\begin{array}{cc}
-0.2712 & 12.8424 \\
0.6434 & 46.3766 \\
0.1172 & -20.5476
\end{array}\right] \text {. }
$$ 


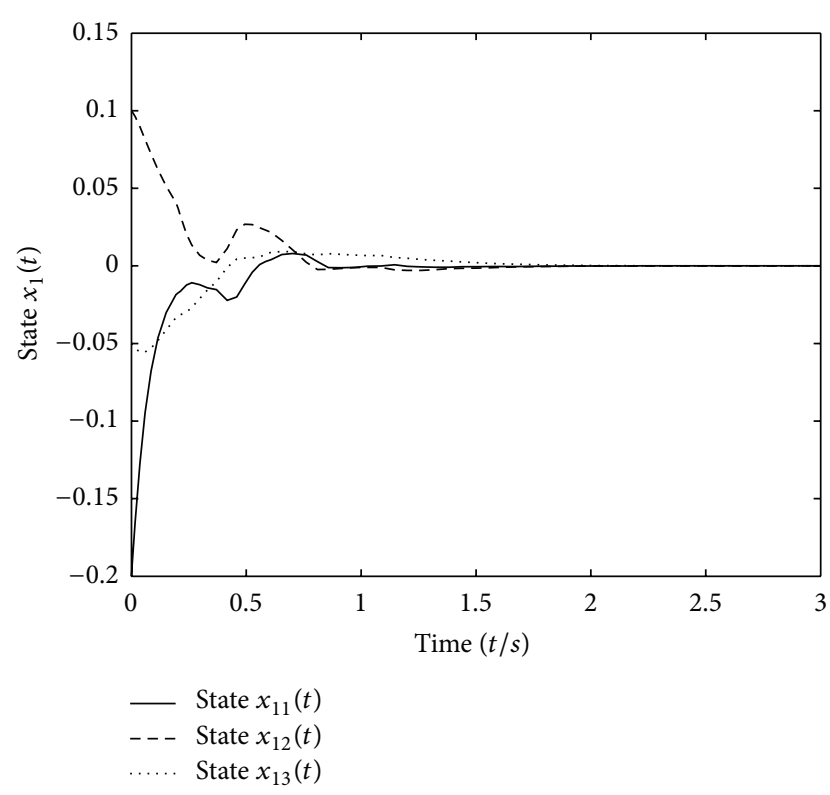

FIGURE 1: State responses of the first closed-loop subsystem.

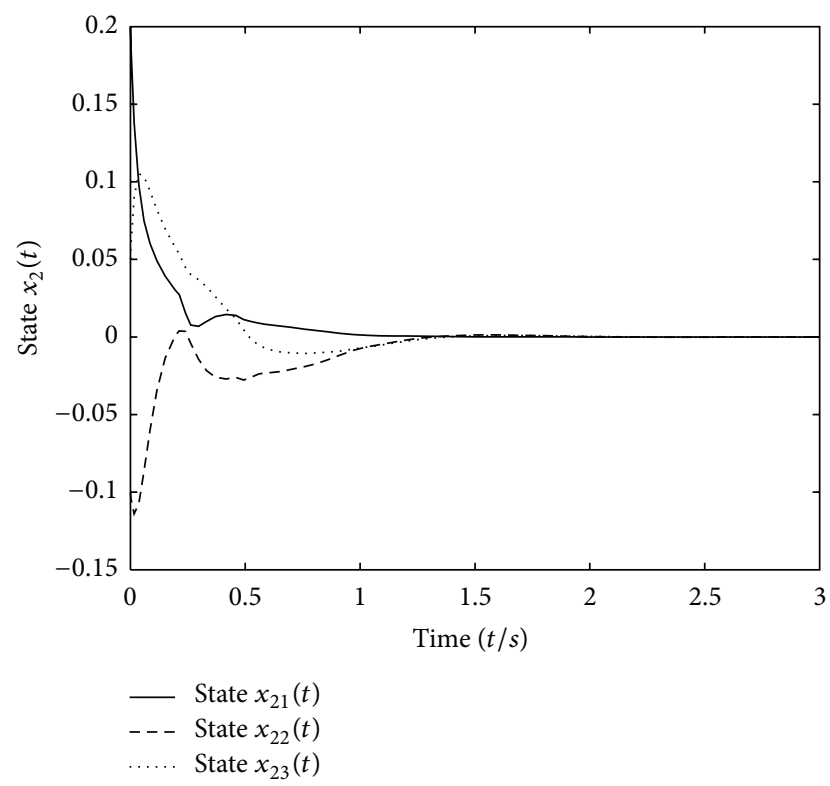

FIGURE 2: State responses of the second closed-loop subsystem.

Under the following initial condition

$$
\phi_{1}(t)=\left[\begin{array}{c}
-0.2 e^{2 t} \\
0.1 \\
0.4 t-0.05
\end{array}\right], \quad \phi_{2}(t)=\left[\begin{array}{c}
0.2 e^{t} \\
0.2 t-0.1 \\
0.05
\end{array}\right],
$$

the simulation results are shown in Figures 1, 2, 3, 4, 5, 6, 7, $8,9,10,11$, and 12 . With the observer-based control applied, the state responses of the closed-loop system are depicted in Figures 1-2. The control signals based on the observer are rather smooth in Figures 3 and 4 . The output signals of the closed-loop system are shown in Figures 5 and 6 . The state responses and the output signals of the observer system (6) are presented in Figures 7-10, respectively. The state responds

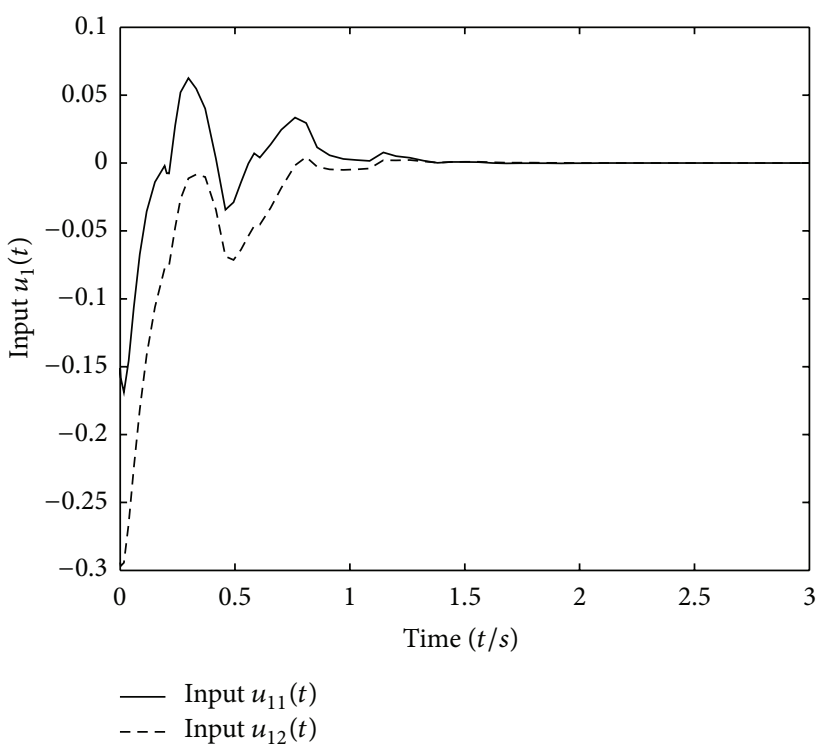

FIGURE 3: The control signals of the first subsystem.

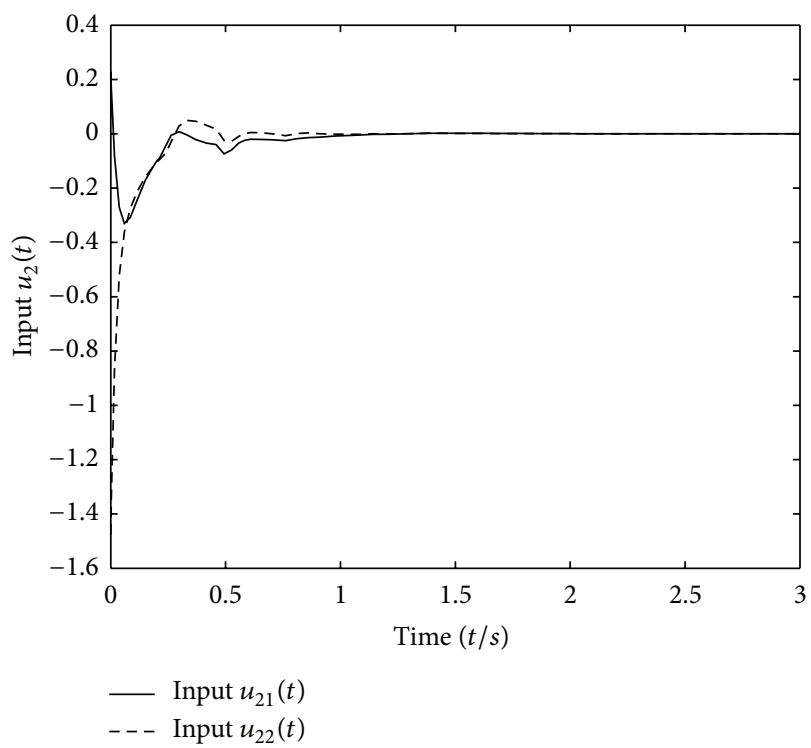

FIGURE 4: The control signals of the second subsystem.

of the error dynamic system (7) are shown in Figures 11 and 12.

The simulations results indicate that the designed observer can stabilize the error dynamic system and estimate the states of the interconnected systems of neutral type.

Remark 7. For this example, $N=2$. When $i=1$, we can obtain $Q_{11}, Q_{12}, \bar{W}_{21}, W_{12}, \bar{G}_{21}$, and $G_{12}$ by solving $\operatorname{LMI} \Omega_{1}<0$ in (11). Furthermore, according to $\bar{W}_{21}=Q_{11} W_{21} Q_{11}$ and $\bar{G}_{21}=Q_{12} G_{21} Q_{12}$, we have $W_{21}$ and $G_{21}$. When $i=2$, inequality $\Omega_{2}<0$ in (11) is not LMI because of the existence of the interconnection matrix $\bar{W}_{12}=Q_{21} W_{12} Q_{21}$ and $\bar{G}_{12}=$ $Q_{22} G_{12} Q_{22}$ (here, $W_{12}$ and $G_{12}$ are constant matrices coming 


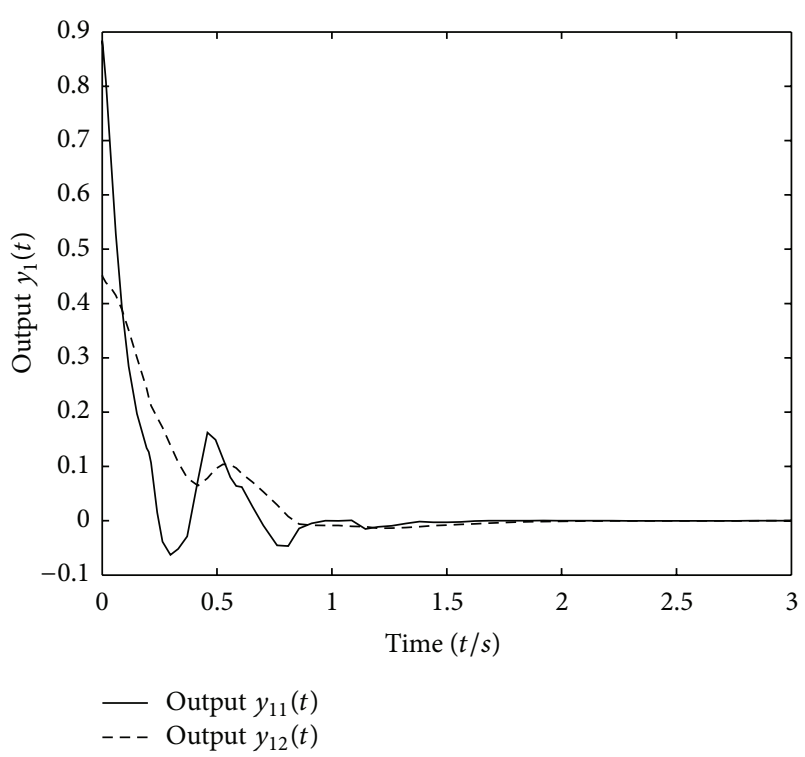

FIGURE 5: Output responses of the first subsystem.

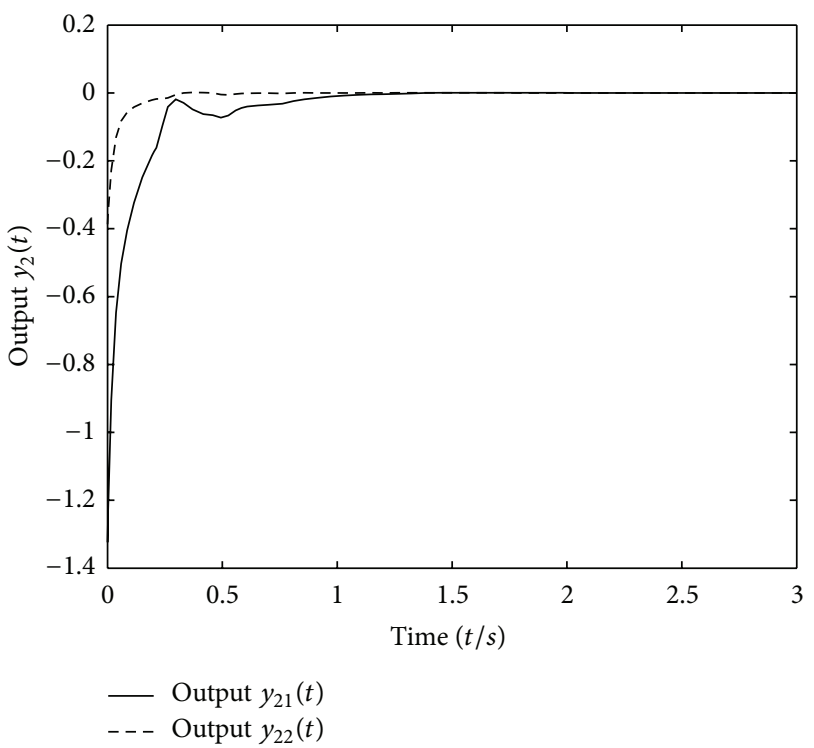

FigURE 6: Output responses of the second subsystem.

from $\Omega_{1}<0$ ). However, the transformed inequality is an LMI after applying Schur complement to $\Omega_{2}<0$. Hence, the controller gain $K_{2}$ and observer gain $L_{2}$ can be obtained by finding feasible set to the transformed inequality of $\Omega_{2}<0$.

\section{Conclusion}

The observer-based decentralized control problem of uncertain interconnected systems of neutral type is complex and challenging. In framework of Lyapunov stability theory, a novel mathematical technique to deal with the parametric disturbances is developed to obtain the sufficient conditions of existing anticipated controller and observer. The sufficient conditions are the coupled LMIs and depend on not only the

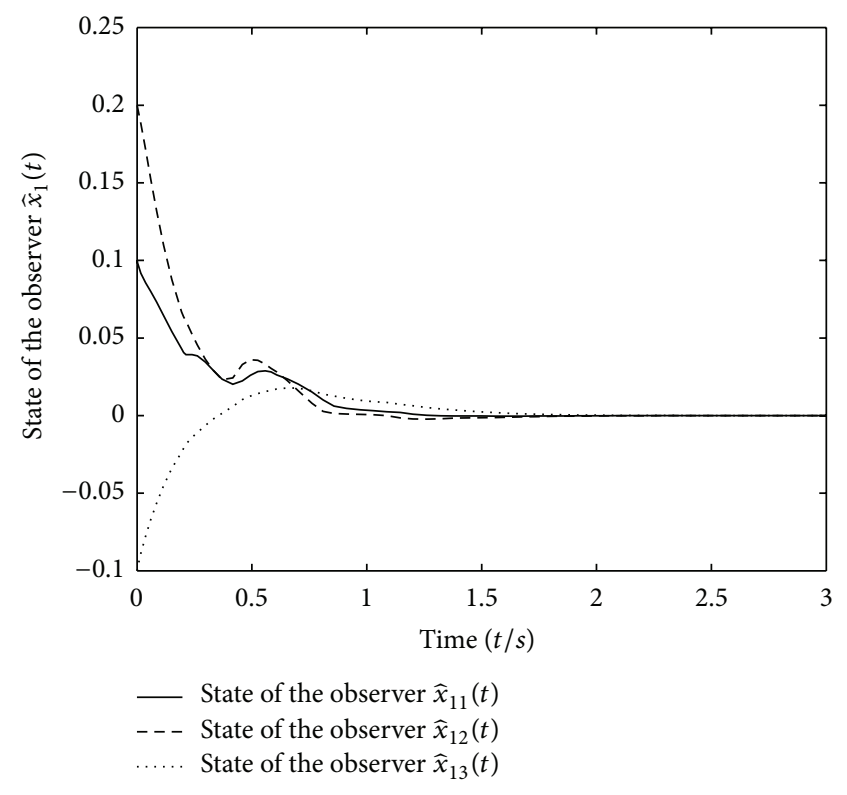

FIGURE 7: State responses of the first observer subsystem.

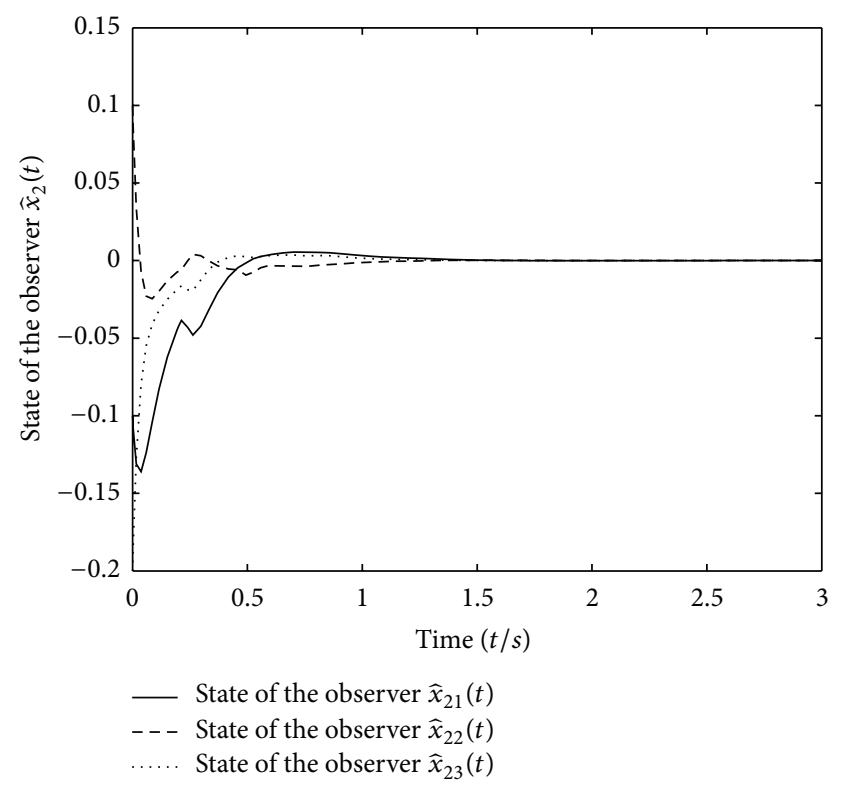

FIGURE 8: State responses of the second observer subsystem.

sizes of delays, but also the information of derivatives. The numerical example and the corresponding simulation results elucidate that the results obtained in this paper are effective.

\section{Acknowledgments}

This research is supported by the Natural Science Foundation of China (no. 61104106), the Science Foundation of Department of Education of Liaoning Province (no. L2012422), and 


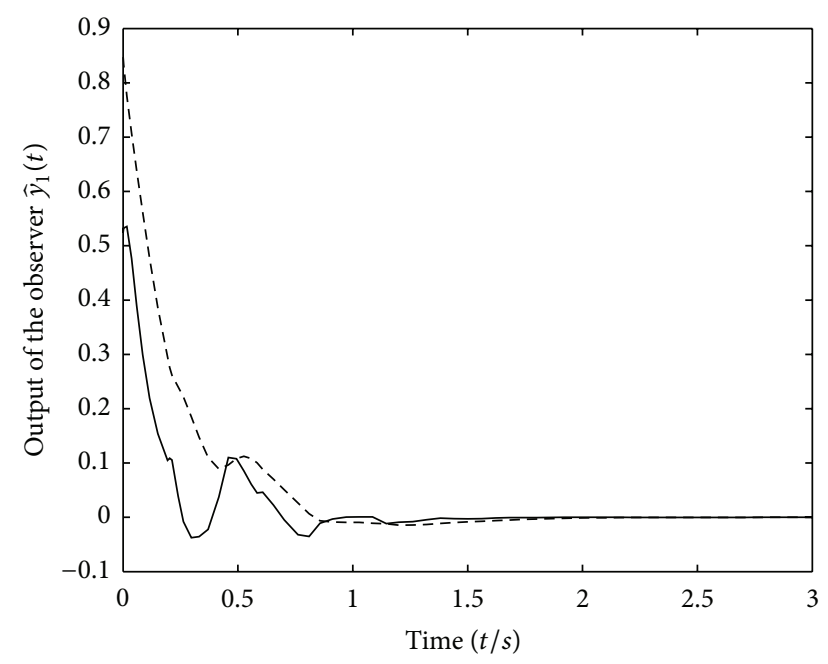

- Output of the observer $\widehat{y}_{11}(t)$

- - Output of the observer $\hat{y}_{12}(t)$

FIGURE 9: Output responses of the first observer subsystem.

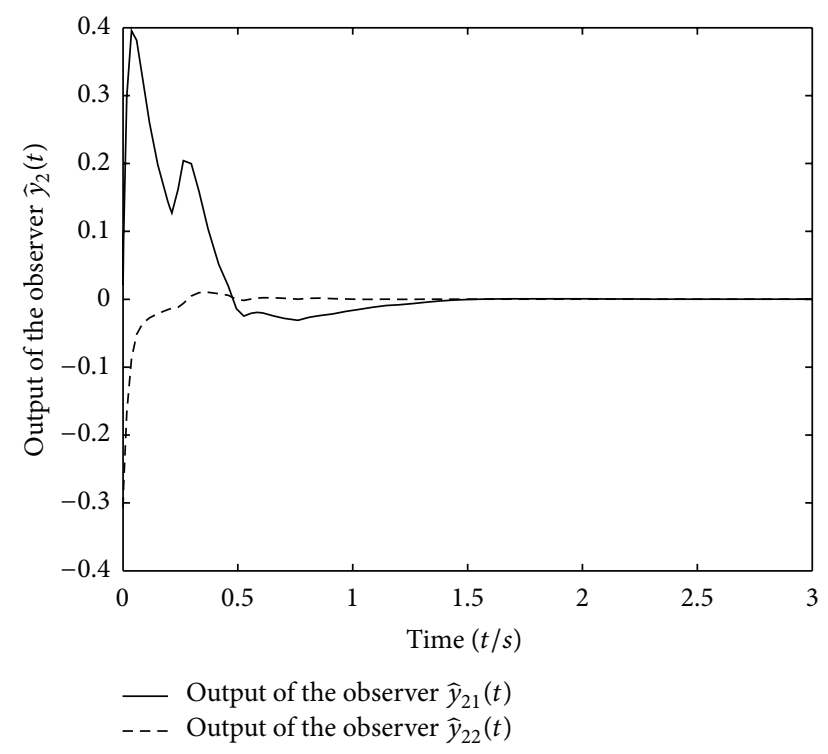

FIGURE 10: Output responses of the second observer subsystem.

the Doctorial Science Foundation of Shenyang University (no. 20212340).

\section{References}

[1] M. S. Mahmoud and Y. Xia, "A generalized approach to stabilization of linear interconnected time-delay systems," Asian Journal of Control, vol. 14, no. 6, pp. 1539-1552, 2012.

[2] X. Ye, "Decentralized adaptive stabilization of large-scale nonlinear time-delay systems with unknown high-frequency-gain signs," IEEE Transactions on Automatic Control, vol. 56, no. 6, pp. 1473-1478, 2011.

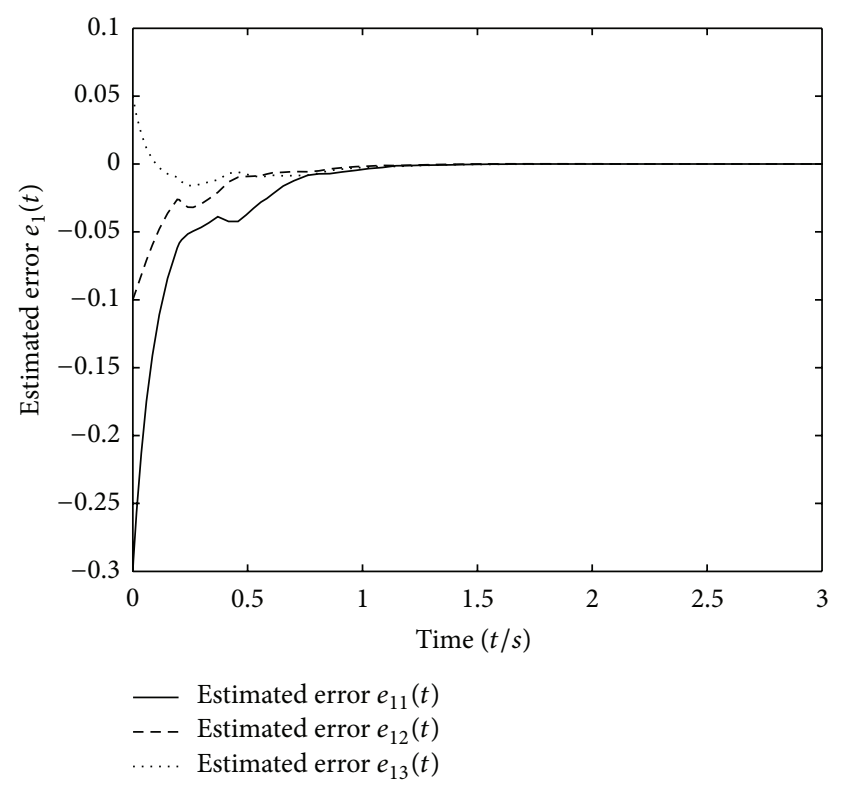

FIGURE 11: State responses of the first error subsystem.

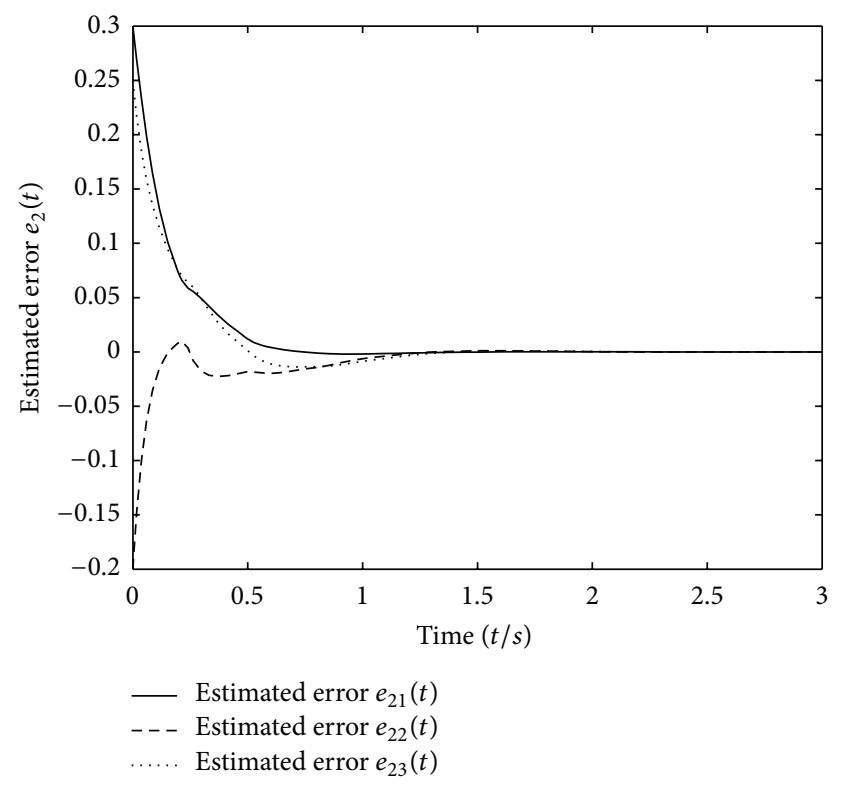

FIGURE 12: State responses of the second error subsystem.

[3] S. J. Yoo, N. Hovakimyan, and C. Cao, "Decentralised $L_{1}$ adaptive control for large-scale non-linear systems with interconnected unmodelled dynamics," IET Control Theory \& Applications, vol. 4, no. 10, pp. 1972-1988, 2010.

[4] X. Ouyang and X. Chen, "Modeling and decentralized control of complex dynamic interconnected systems," International Journal of Information \& Systems Sciences, vol. 5, no. 2, pp. 248259, 2009.

[5] K. Kalsi, J. Lian, and S. H. Źak, "Decentralized dynamic output feedback control of nonlinear interconnected systems," IEEE Transactions on Automatic Control, vol. 55, no. 8, pp. 1964-1970, 2010. 
[6] M. S. Mahmoud, "Decentralized stabilization of interconnected systems with time-varying delays," IEEE Transactions on Automatic Control, vol. 54, no. 11, pp. 2663-2668, 2009.

[7] G. Zhai, N. Chen, and W. Gui, "A study on decentralized $H_{\infty}$ feedback control systems with local quantizers," Kybernetika, vol. 45, no. 1, pp. 137-150, 2009.

[8] S. K. Yee and J. V. Milanović, "Fuzzy logic controller for decentralized stabilization of multimachine power systems," IEEE Transactions on Fuzzy Systems, vol. 16, no. 4, pp. 971-981, 2008.

[9] R. Garcia-Hernandez, E. N. Sanchez, E. Bayro-Corrochano, M. A. Llama, and J. A. Ruz-Hernandez, "Real-time decentralized neural backstepping control: application to a two DOF robot manipulator," International Journal of Innovative Computing, Information and Control, vol. 7, no. 2, pp. 965-976, 2011.

[10] Y. Xu and S. Hu, "The existence and uniqueness of the solution for neutral stochastic functional differential equations with infinite delay in abstract space," Acta Applicandae Mathematicae, vol. 110, no. 2, pp. 627-638, 2010.

[11] H. Mukaidani, "Decentralized stochastic guaranteed cost control for uncertain nonlinear large-scale interconnected systems under gain perturbations," in Proceedings of the American Control Conference (ACC '09), pp. 5097-5102, St. Louis, Mo, USA, June 2009.

[12] V. Kolmanoskii and A. Myshkis, Applied Theory of Functional Differential Equations, Kluwer Academic Publishers, Dodrecht, The Netherlands, 1992.

[13] R. K. Brayton, "Bifurcation of periodic solutions in a nonlinear difference-differential equations of neutral type," Quarterly of Applied Mathematics, vol. 24, pp. 215-224, 1966.

[14] Y. Kuang, Delay Differential Equations with Applications in Population Dynamics, vol. 191 of Mathematics in Science and Engineering, Academic Press, Boston, Mass, USA, 1993.

[15] S. Niculescu, Delay Effects on Stability: A Robust Control Approach, vol. 269 of Lecture Notes in Control and Information Sciences, Springer, Berlin, Germany, 2001, A robust control approach.

[16] P. Balasubramaniam, R. Krishnasamy, and R. Rakkiyappan, "Delay-dependent stability of neutral systems with timevarying delays using delay-decomposition approach," Applied Mathematical Modelling, vol. 36, no. 5, pp. 2253-2261, 2012.

[17] R. Rakkiyappan, P. Balasubramaniam, and R. Krishnasamy, "Delay dependent stability analysis of neutral systems with mixed time-varying delays and nonlinear perturbations," Journal of Computational and Applied Mathematics, vol. 235, no. 8, pp. 2147-2156, 2011.

[18] X. Nian, H. Pan, W. Gui, and H. Wang, "New stability analysis for linear neutral system via state matrix decomposition," Applied Mathematics and Computation, vol. 215, no. 5, pp. 18301837, 2009.

[19] W. B. Ma, N. Adachi, and T. Amemiya, "Delay-independent stabilization of uncertain linear systems of neutral type," Journal of Optimization Theory and Applications, vol. 84, no. 2, pp. 393405, 1995.

[20] Q. Han, X. Yu, and K. Gu, "On computing the maximum time-delay bound for stability of linear neutral systems," IEEE Transactions on Automatic Control, vol. 49, no. 12, pp. 22812285, 2004.

[21] K. Moezzi and A. G. Aghdam, "Adaptive robust control of uncertain neutral time-delay systems," in Proceedings of the American Control Conference (ACC '08), pp. 5162-5167, Seattle, Wash, USA, June 2008.
[22] K. Zhang, B. Jiang, and A. Shumsky, "A new criterion of fault estimation for neutral delay systems using adaptive observer," Acta Automatica Sinica, vol. 35, no. 1, pp. 85-91, 2009.

[23] L. Huang and X. Mao, "Delay-dependent exponential stability of neutral stochastic delay systems," IEEE Transactions on Automatic Control, vol. 54, no. 1, pp. 147-152, 2009.

[24] R. Yang, P. Shi, G.-P. Liu, and H. Gao, "Network-based feedback control for systems with mixed delays based on quantization and dropout compensation," Automatica, vol. 47, no. 12, pp. 2805-2809, 2011.

[25] X. Su, P. Shi, L. Wu, and Y.-D. Song, "A novel control design on discrete-time Takagi- Sugeno fuzzy systems with time-Varying delays," IEEE Transactions on Fuzzy Systems, 2012.

[26] X. Su, P. Shi, L. Wu, and Y. -D. Song, "A novel approach to filter design for T-S fuzzy discrete-time systems with time-varying delay," IEEE Transactions on Fuzzy Systems, vol. 20, no. 6, pp. 1114-1129, 2012.

[27] D. Zhang, H. Wang, B. Lu, and Z. Wang, "LMI-based fault detection fuzzy observer design with multiple performance constraints for a class of non-linear systems: comparative study," International Journal of Innovative Computing, Information and Control, vol. 8, no. 1 B, pp. 633-645, 2012.

[28] R. B. Messaoud, N. Zanzouri, and M. Ksouri, "Local feedback unknown input observer for nonlinear systems," International Journal of Innovative Computing, Information and Control, vol. 8, no. 2, pp. 1145-1154, 2012.

[29] D. W. C. Ho and G. Lu, "Robust stabilization for a class of discrete-time non-linear systems via output feedback: the unified LMI approach," International Journal of Control, vol. 76, no. 2, pp. 105-115, 2003.

[30] P. Gahinet, A. Nemirovski, A. J. Laub, and M. Chilali, LMI Control Toolbox User's Guide, The Math Works, Natick, Mass, USA, 1995. 


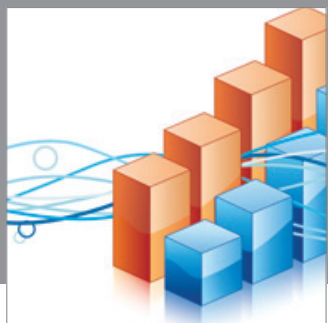

Advances in

Operations Research

mansans

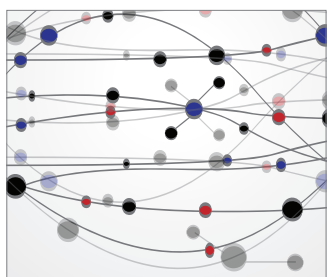

The Scientific World Journal
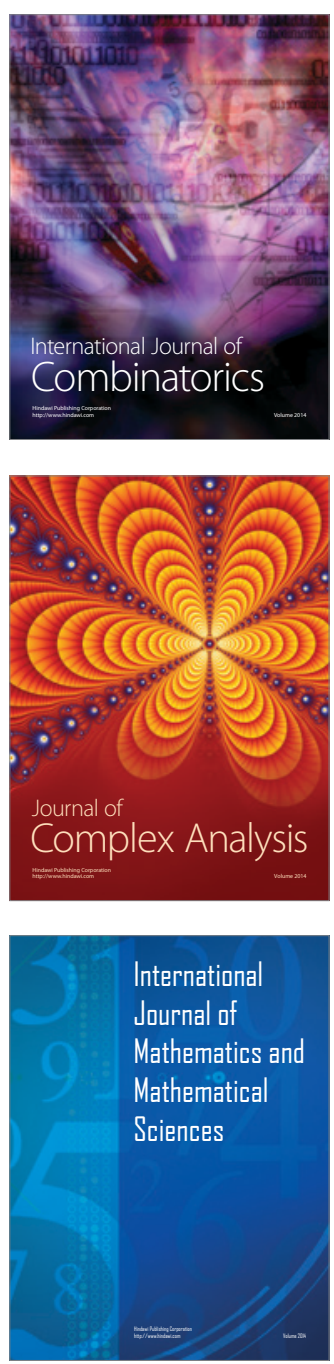
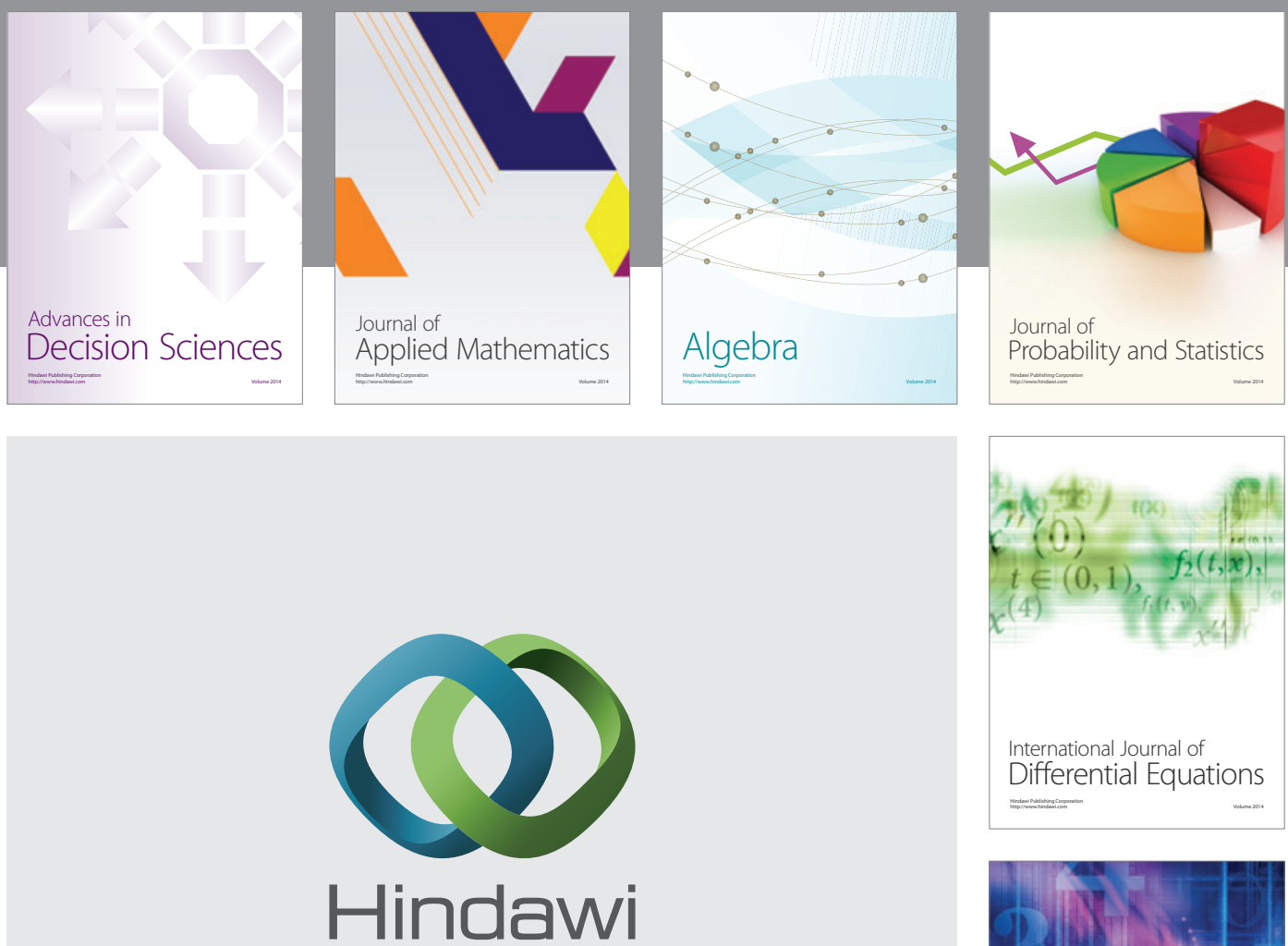

Submit your manuscripts at http://www.hindawi.com
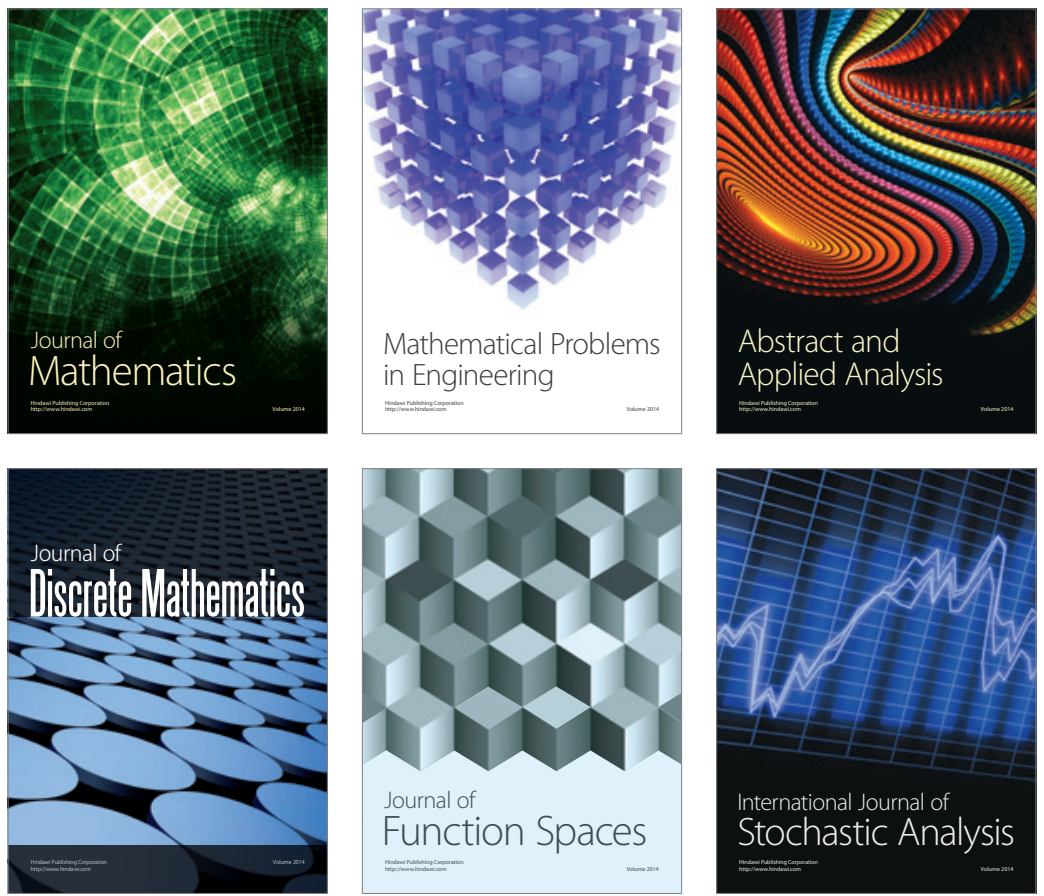

Journal of

Function Spaces

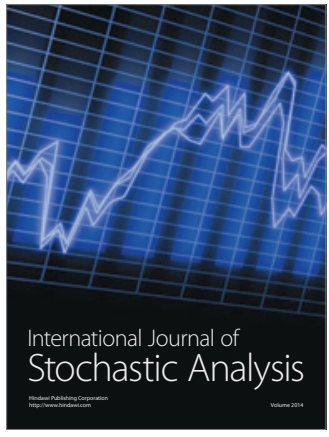

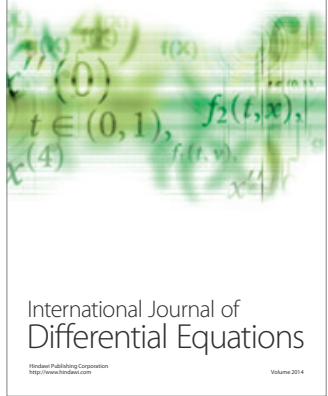
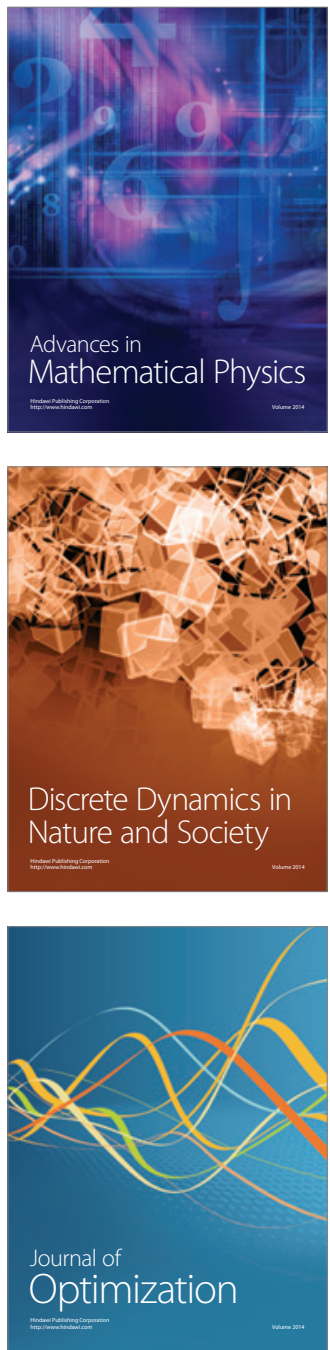